\title{
The Analysis of Moment Characteristics of Variable Forward-swept Wing Mechanism with a Double Slideway
}

\author{
XinbingSu ${ }^{a}$, HaoyangFeng ${ }^{b}$, BinlinMa $^{c}$, XuWang $^{d}$ \\ Institute of Aeronautics and Astronautics Engineering, Air Force Engineering University, $\mathrm{Xi}^{\prime}$ an \\ 710038, China \\ a sxinbing@sohu.com, ${ }^{b} 78318852 @ q q . c o m,{ }^{c} 171430198 @ q q . c o m,{ }^{d}$ wangxu01@126.com
} Keywords: variable forward-swept wing, switching mechanism,double slideway, moment
characteristics.

\begin{abstract}
Based on the variable forward-swept wing configuration, this paper utilizes a variable forward-swept wing mechanism with a double slideway, which makes it possible for the aircraft to switch among orthogonal wing, forward-swept wing and delta wing freely. The general configuration of the variable forward-swept wing mechanism with a double slideway is elucidated by means of a three-dimensional model plot and then the mathematic model is also established. The motion characteristics of linear motion are simulated, analyzed and optimized. The results indicate that the variable forward-swept wing mechanism with a double slideway can meet the aerodynamic requirements better. Moreover, there exists a larger moment amplification factor in forward-swept small Angle to provide greater control driving moment. Therefore, this mechanism may serve as a useful reference when developing a morphing aircraft.
\end{abstract}

\section{Introduction}

The variable forward-swept wing (VFSW) aircraft is a typical variant aircraft. Based on different mission requirements and flight environments, it can free change among straight wing, forward-swept wing and delta wing by changing the angle of forward-swept. It combines pneumatic advantages and variation characteristics of the three wings, to suitable for different tasks. The variable forward-swept wing mechanism technology is a key technology of morphing aircraft. Its structure not only needs to meet the demands of force and moment caused by wing lift, drag and switching wing driving force, but also needs to decrease its mass as far as possible. Nowadays, domestic and overseas researches almost stay in the stage of concept designing[1-3] with few of reports of variable forward-swept wing mechanism. Traditional VFSW mechanisms are divided into shaft type and four-bar linkage type[4-7]. The former is easy to realize, but has excessively centralized load at body-wing connections and has large travel of aerodynamic center when switching wing. The latter can change vertical and lateral position when forward-swept angle is changed, but forward-swept angle is limited on some degree and the four-bar linkage has uncompact structure and low loading capability. Overseas variable backward-swept wing aircrafts change angles through shaft type variable wing mechanism. [8]

Combined with the three typical configurations of VFSW, the study found in the changing from straight wing to delta wing, the wing sweep Angle is changing, and the whole wing is moving back. If the wing uses the traditional shaft institution, there are five problems as follows: (1) The wing can't move back with forward-swept Angle change, it is difficult to reach the delta wing layout; (2) If the axis point is located near the leading edge of wing root, the airframe back-end will naked because wing moves forward. (3) If the axis point is located near the trailing edge of the wing root, increased torque is bound to thickening the rotation point and the delta wing trailing edge is not sharp. (4) In the process of deformation, the aircraft longitudinal stability margin was changed due to the movement of aerodynamic center. (5) Forward-swept wing layout is benefit for using short neck coupled canard layout, the optimization of aerodynamic characteristics is restricted due to the wing rotating around a fixed axle. Comprehensive consideration, for the VFSW, the traditional 
rotor wing body moving mechanism unable to meet the requirements of deformation, and can't keep ideal aerodynamic shape in different forward-swept Angle.

Based on the design of VFSW mechanism with a double slideway of CATIA, the mathematic model is established and the moment characteristics of linear slideway are simulated, analyzed and optimized. As a kind of referential scheme of morphing aircraft designing, above discussions are used to analyze its moment characteristics. Compared with traditional mechanisms, this mechanism can change vertical and lateral position when the wing is changed, making the deformation of aerodynamic layout without limitation of rotor position. It has many advantages such as strong loading capability, large movable wing area, fluent movement and the best comprehensive performance. The optimal comprehensive performance of design can be achieved in whole process of switching wing[9].

\section{The brief introduction of variable forward-swept wing mechanism with a double slideway[10]}

The whole design of Wing body muscle system as seen in Figure1.(1)body; (2)wing; (3)slideway; (4) ball screw; (5)slider; (6) driving rod.The process of switching wing was driving by electrical machine. The electrical machine drives ball screw to rotate by use of coupler. Ball screw pair, which is combination of ball screw and nut, changes the movement of nut from rotation to linear motion. Nut drives right and left active slider to slide on each slideway by use of two driving rods. In the meantime, right and left driven sliders slide on each slideway, respectively. So, as to one side of wings, change of relative position of two wing-body connections means change of angle of forward-swept wing. Therefore, the forward-swept wing movement law can be found by the following geometric relationship: the distance between two wing-body connections, geometrical configuration of slideways, and the relative position between two slideways.
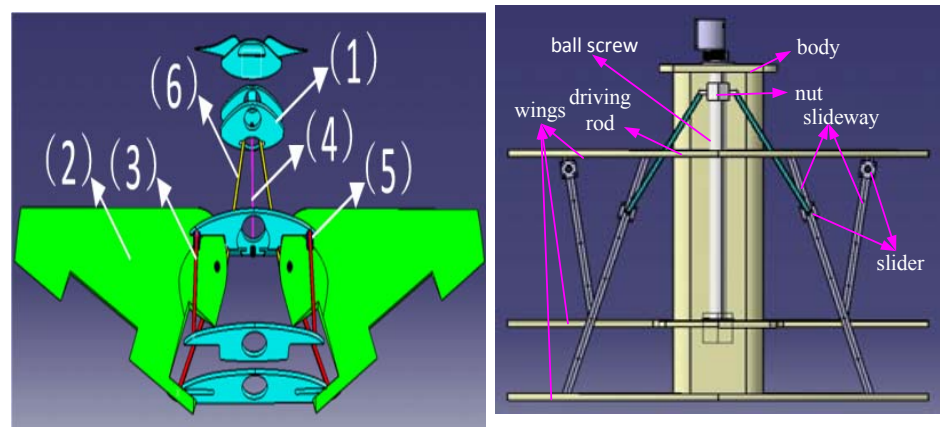

Fig 1. The variable forward-swept wing mechanism

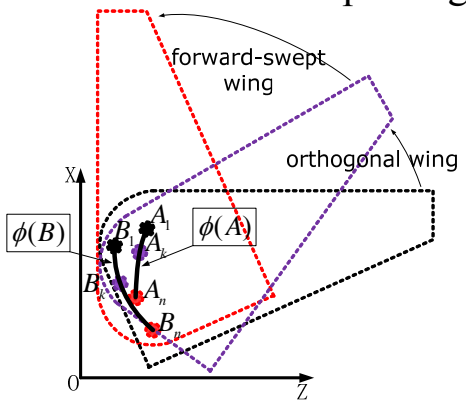

Fig 2. Schematic diagram of double slideway

Sweep Angle is an important parameter impact of the VFSW configuration and dynamic characteristics. On movable plane, point $A, B$ can uniquely determine the position of wing. As seen in Figure 2, taking right wing as an example, in Cartesian coordinate system $0 x z$ on wing plane, under a certain typical flight state $k(k=2,3 \ldots n-1)$ with the best aerodynamic configuration, denote two fixed wing points as $A_{k}\left(x_{A k}, Z_{A k}\right), B_{k}\left(x_{B k}, z_{B k}\right)$, respectively. Connect all $A, B$ with all states from $1 \sim k \sim n$ by smooth curve, then we get two curves $\phi(A), \phi(B)$, which describe geometric shapes and spatial position of slideways and reflect the forward-swept wing movement law when changing wing. 


\section{Establish mathematic model}

Taking the tail end of aircraft as origin, coordinate system oxz is established. The horizontal axis is along the spanwise and vertical axis coincides with body axis. According to aerodynamic configuration of three typical layouts of VSWF aircraft, fitting of discrete points define four trajectories: $\phi(A), \phi(B), \phi(C), \phi(D)$ where $A, B$ are fixed points on wing and $C, D$ are two endpoints of driving rods. As seen in Figure 3, the state parameters of each part of double slideway mechanism in some states.
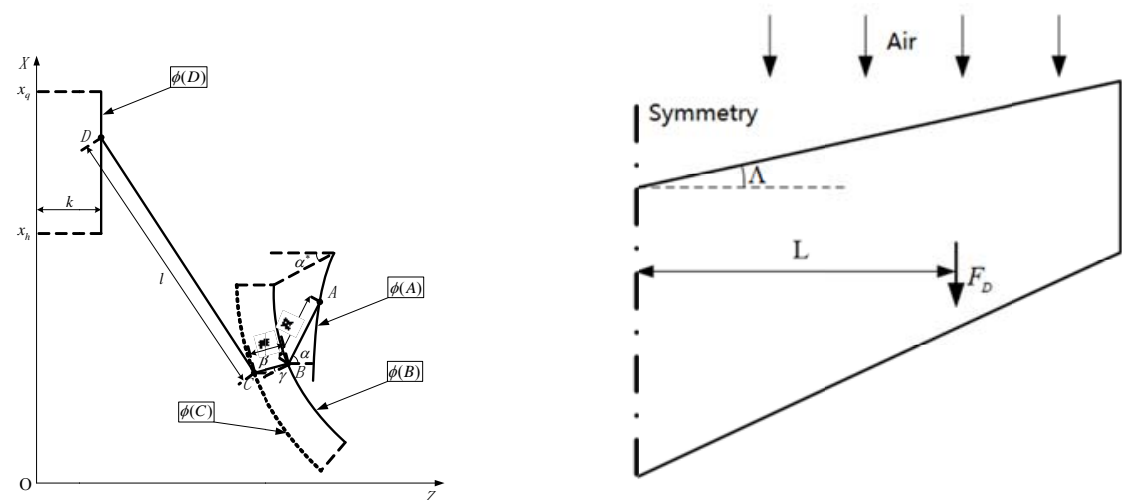

Fig 3. Principle diagram of double slidewaymechanism Fig 4. The drag of wing in deformation

Denote coordinates of $A, B, C, D$ as $\left(z_{A}, x_{A}\right) 、\left(z_{B}, x_{B}\right) 、\left(z_{C}, x_{C}\right) 、\left(z_{D}, x_{D}\right)$; Denote $\alpha$ as angle between $\mathrm{AB}$ and $\mathrm{OZ}$ axis, $\beta$ as angle between $\mathrm{BC}$ and $\mathrm{OZ}$ axis, respectively. Then denote $\gamma$ as angle between $\mathrm{BC}$ and normal direction of $\mathrm{B}$ on curve (A), $\Lambda$ as forward-swept wing angle. $\mathrm{AB}=\mathrm{n}, \mathrm{BC}=\mathrm{m}, \mathrm{CD}=1$, Screw coincides with $\mathrm{OX}$ axis. $\mathrm{K}$ is the distance from driveshaft forward terminal to screw. In this mechanism, coordinates of $A, B, C, D$ satisfy the Equation 1.

$$
\left\{\begin{array}{l}
x_{A}=f_{A}\left(z_{A}\right), \quad x_{B}=f_{B}\left(z_{B}\right), \quad z_{D}=k, x_{D} \in\left[x_{h}, x_{q}\right] \\
\left(z_{A}-z_{B}\right)^{2}+\left(x_{A}-x_{B}\right)^{2}=n^{2} \\
\left(z_{B}-z_{C}\right)^{2}+\left(x_{B}-x_{C}\right)^{2}=m^{2} \\
\left(z_{C}-z_{D}\right)^{2}+\left(x_{C}-x_{D}\right)^{2}=l^{2} \\
\arctan \frac{\left|x_{B}-x_{C}\right|}{\left|z_{B}-z_{C}\right|}+\arctan \left|f_{B}^{\prime}\left(z_{B}\right)\right|=\frac{\pi}{2}-\gamma
\end{array}\right.
$$

Where $\mathrm{n}, 1$ and $\mathrm{m}$ are the length of $\mathrm{AB}, \mathrm{BC}$ and $\mathrm{CD} \cdot \mathrm{k}$ is the distance firom driving rod front point to screw.

In order to simplify analysis, we replace all parameters in Equation 1 lby functions of input parameter $\mathrm{x}$ (coordinate of nut on $\mathrm{OX}$ axis). Then we get following Equations.

$$
\begin{aligned}
& s_{t}=f_{s t}(x, k, l, m, n, \gamma) ; \quad s=z, x ; t=A, B, C, D \\
& \Lambda=\alpha-\alpha^{*}=\arctan \frac{\left|x_{A}-x_{B}\right|}{\left|z_{A}-z_{B}\right|}-\alpha^{*}=f_{\Lambda}(x, k, l, m, n, \gamma) \\
& \beta=\frac{\pi}{2}-\arctan \left|f_{B}^{\prime}\left(z_{B}\right)\right|-\gamma=f_{\beta}(x, k, l, m, n, \gamma)
\end{aligned}
$$

Considering the requirements of motion characteristics in the process of deformation, moment transmit characteristics and structure space of deformation mechanism for VFSW, the feature parameters of double slide rail type wing body moving mechanisms usually need to optimized. When the aerodynamic shape of various configurations were optimized, the position of A, B were determined by the requirements of intensity, and the $\phi(A), \phi(B)$ were determined by Curve fitting of discrete points. When $\phi(A), \phi(B)$ are determined, $\mathrm{n}$ is a Constant value, optimization can be made by adjusting $x_{q}, x_{h}, k, l, m, \gamma$. 
One of primary problems which should be considered in wing-body moving mechanism design process is whether the enough driving moment could be produced for overcoming the air resistance when the wing swept is under high-speed flight.

In preliminary analysis of double slide rail type wing body moving mechanism, the friction force of screw, slippery course, and shaft friction were ignored. Sets the output power of motor as $\mathrm{W}$, the driving force on the screw as $\mathrm{F}$, Nut on the screw axis direction of displacement as $\mathrm{S}$, motor driver on the wing of overcome the air resistance moment as M, sweep-forward wing Angle changes within a certain time as $\theta$. When determine a transient state as the research object, according to the energy conservation, we can know that,

$$
\begin{gathered}
W=F \cdot S=2 M \cdot \theta \\
\text { namely: } \quad F \cdot v \cdot \Delta t=2 M \cdot \omega \cdot \Delta t \Rightarrow F \cdot \frac{d x}{d t} \cdot \Delta t=2 M \cdot \frac{d \Lambda}{d t} \cdot \Delta t \Rightarrow F \cdot d x=2 M \cdot d \Lambda \\
\text { so, } M=\frac{F}{2} \cdot \frac{d x}{d \Lambda}=\frac{F}{2} \cdot\left(\frac{d \Lambda}{d x}\right)^{-1}=\frac{F}{2} \cdot\left[f_{\Lambda}^{\prime}(x, k, l, m, \gamma)\right]^{-1}
\end{gathered}
$$

When the wing deformation in small swept-forward Angle $\Lambda \in\left[0 \sim \Lambda_{e}\right]\left(\Lambda_{e} \in(0 \sim \pi / 2)\right)$, Wing windward area and moment arm both are large, leading to the needed moment become bigger in the change of driving sweep-forward Angle, as seen in Figure4.

After $\Lambda_{e}$ was given, $x_{q}, x_{e}$ were determined by equation (1).

$$
\left\{\begin{array} { l } 
{ f _ { \Lambda } ( x _ { q } , k , l , m , \gamma ) = 0 } \\
{ f _ { \Lambda } ( x _ { e } , k , l , m , \gamma ) = \Lambda _ { e } }
\end{array} \Rightarrow \left\{\begin{array}{l}
x_{q}=f_{x q}(k, l, m, \gamma) \\
x_{e}=f_{x e}(k, l, m, \gamma)
\end{array}\right.\right.
$$

It is on the premise that enough driving moment is satisfied, in order to reduce the power of motor, VFSW mechanism should have a small transmission ratio within the scope of sweep-forward angle $0 \sim \Lambda_{e}$. The transmission ratio was judged by the objective function $P$.

$$
P\left(x_{q}, x_{e}, k, l, m, \gamma\right)=\int_{x_{e}}^{x_{q}}\left|f_{\Lambda}^{\prime}(x, k, l, m, \gamma)\right| d x \Rightarrow P(k, l, m, \gamma)=\int_{f_{x e}(k, l, m, \gamma)}^{f_{x q}(k, l, m, \gamma)}\left|f_{\Lambda}^{\prime}(x, k, l, m, \gamma)\right| d x
$$

The Movement characteristics of wing deforming, moment transmission characteristics of variable forward-swept wing mechanism and the structure space of mechanism should be considered on design. The values of $k, l, m, \gamma$ were optimized under the condition of equation (1) to get the minimum value of objective function $P(k, l, m, \gamma)$, in order to get small transmission ratio.

\section{Calculation examples}

Because the VFSW aerodynamic shape optimization design is not yet completed in the primary stage, considering the processing difficulty of curve track, two curves $\phi(A), \phi(B)$ can be replaced by straight lines at prophase under the condition of aerodynamic configuration.

(1) Definition $\Lambda$ and derivative of $\Lambda$ with respect to $\mathrm{x}$

The position of A,B can be determined by design requirement of wing-body connection point, and two straight lines can be obtained. It is observed that abscissa $Z$ of point $A$ rarely changes. Double slideway wing-body mechanism can be simplified for increasing the processing difficulty. The trajectory of $\mathrm{A}$ is parallel with $\mathrm{X}$ axis by adjusting its position. So, coordinates of $A, B, C, D$ can be simplified into following pattern.

$$
\left\{\begin{array}{l}
z_{A}=a, \quad x_{B}=b z_{B}+c, \quad x_{C}=b z_{C}+d ; \quad d=c-m \cdot \cos \gamma \sqrt{1+b^{2}} \\
z_{D}=k, x \in\left[x_{h}, x_{q}\right] \\
\left(z_{A}-z_{B}\right)^{2}+\left(x_{A}-x_{B}\right)^{2}=n^{2} \\
\left(z_{B}-z_{C}\right)^{2}+\left(x_{B}-x_{C}\right)^{2}=m^{2} \\
\left(z_{C}-z_{D}\right)^{2}+\left(x_{C}-x_{D}\right)^{2}=l^{2}
\end{array}\right.
$$


The design parameters of variable forward-swept wing mechanism with a double slideway (in millimeters) are as followings. After simplifying, we combine the boundary dimension of Technical Demonstration and requirement of load ability of wing-body joints. The design parameters of wing-body moving mechanism were determinedprimarily, as follows, $a=168, b=-1.732, c=481.673, d=447.673, k=25, l=289.691, m=18.366, \gamma=22.236^{\circ}, n=72.056$ ,$\alpha^{*}=30^{\circ}$. Where $a, b, c, n, \alpha^{*}$ are all constant.

The position of A, B, C, D were determined by equation (9). $\Lambda$ andderivative of $\Lambda$ with respect to $\mathrm{x}$ were determined by A,B. $x_{q}, x_{h}$ were determined by $\Lambda_{\text {straight }}=f_{\Lambda}\left(x_{q}, k, l, m, \gamma\right)=0$, $\Lambda_{\text {delta }}=f_{\Lambda}\left(x_{h}, k, l, m, \gamma\right)=\pi / 2$.

(2) Driving moment transmit characteristics

The relationship between torque $M$ for overcoming aerodynamic resistance on the wing and output torque $M_{\mathrm{sc}}$ of motor can be determined by equation (6). Determine output torque of motor as $M_{\mathrm{sc}}$, revolving speed as $n_{r}$ ball-screw travel as $\lambda$, and $K=M / M_{\mathrm{sc}}$.

$$
\begin{gathered}
F \cdot v=M_{\mathrm{sc}} \cdot \omega \Rightarrow F \cdot n_{r} \cdot \lambda=M_{\mathrm{sc}} \cdot n_{r} \cdot(2 \pi) \Rightarrow F=\frac{2 \pi M_{\mathrm{sc}}}{\lambda} \\
\text { So, } K=\frac{M}{M_{\mathrm{sc}}}=\frac{\pi}{\lambda} \cdot\left[f_{\Lambda}^{\prime}(x, k, l, m, \gamma)\right]^{-1}
\end{gathered}
$$

Under the primary design parameter, the change of $K$ with $\bar{x}$ are seen as figure 5 . It is can be shown that the moment amplification coefficient become small because large transmission ratio under the condition of small forward-swept angle.
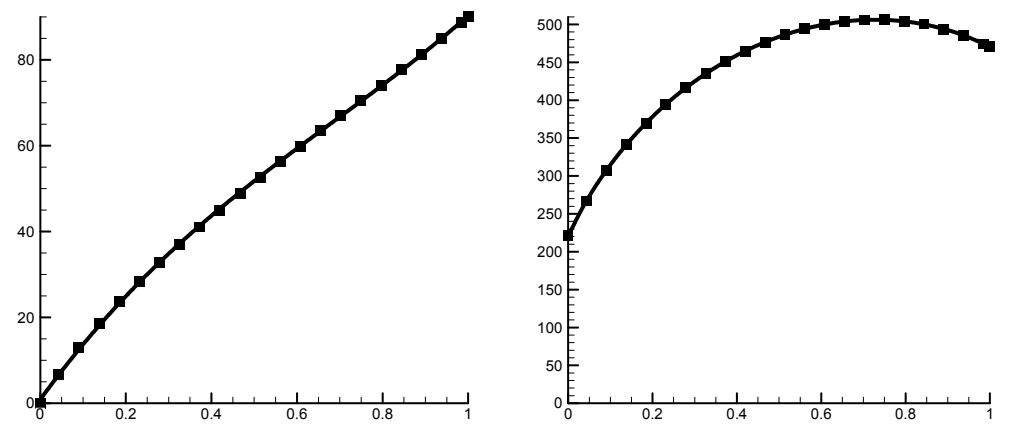

Figure 5. The variation trend of $K_{\text {with }} \bar{x}$

$k, l, m, \gamma$ were optimized by objective function $P(k, l, m, \gamma)$, and then the value of objective function $P(k, l, m, \gamma)$ with range of forward-swept angle $0 \sim \Lambda_{e}$ get the minimum value. $\Lambda_{e}=40^{\circ}, f_{x e}(k, l, m, \gamma)$ can be obtained by equation (7).

After calculation, $k^{*}=13.795, l^{*}=363.687, m^{*}=21.056, \gamma^{*}=28.041^{\circ}$ The variation trend of $K$ with $\bar{x}$ are seen in figure 6 .
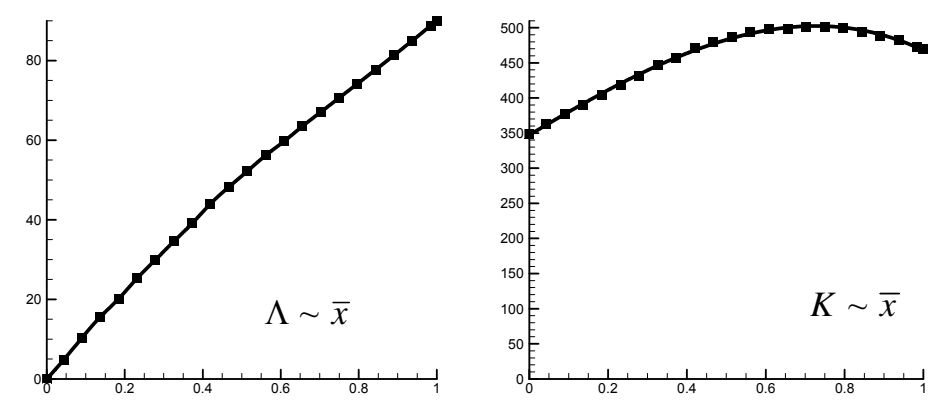

Figure 6 The variation trend of $K$ with $\bar{x}$ after optimization

After optimization, the moment amplification factor $\mathrm{K}$ is increasing from 225 to about 350 in the range of small sweep forward angle. Because aerodynamic resistance is large under small 
sweep forward angle, the result is benefit to double slideway forward-swept mechanism provides large driving moment for overcoming aerodynamic resistance.

\section{Conclusions}

1)This paper analyzes and calculates the moment characteristics of variable forward-swept wing mechanism with a double slideway, establishes its mathematic model and supplies analysis and calculation of some important parameters. Through these methods, we can easily arrange position of mechanism reasonably, design some main parameters, and analyze its moment characteristics using model building.

2)Under the help of simulation calculation and optimization, the moment characteristics of forward-swept wing, which changes with position of nut, was obtained. So, we naturally prove the effectiveness and rationality of these methods.

3)The moment amplification factor $\mathrm{K}$ is increasing in the range of small sweep forward angle. It is benefit to provide large driving moment for overcoming aerodynamic resistance.

Double slide rail type variable wing mechanism has no fixed axis. Through the reasonable design of geometry and spatial location, it can realize arbitrary change of the wing position in theory. Variable wing physical model test shows that the double slide rail type variable wing mechanism can meet the requirements of variable wing aircraft pneumatic layout changes. Compared with traditional deforming wing mechanism, Double slide rail type variable wing mechanism can change the horizontal and lengthwise positions with the wing deformation. The change of aerodynamic configuration is out of limitation of Rotor position. The optimized combination property of aerodynamic configuration can be achieved under any state. Generally speaking, variable forward-swept wing mechanism with a double slideway in this paper can be seen as a useful reference in designing and studying a morphing aircraft.

\section{References}

[1]. Brett A N, Robert L S. Classical Flight Dynamics of A Variable Forward-Sweep-Wing Aircraft. Journal of Guidance, 1986, 9(2): 352-362.

[2].Northrop Grumman Corporation. Aircraft with Variable Forward Sweep Wing: USA, US5984231. 1999.

[3]. Shigeru Horinouchi. Variable Forward Swept Wing Supersonic Aircraft Having Both Low-Boom Characteristics and Low-Drag Characteristics: USA, US Fresh Patent 20050230531. 2005.

[4]. Wang X, Liu W F, Mi K. A Design of Wing-Body Revolved Mechanism for UAV with Variable Forward-Swept Wing. Journal of Air Force Engineering University: Natural Science Education, 2009, 10(3): 1-4.

[5]. Wang D Z, Dong E B, Xu M. Structural Design and Kinematics Simulation for t he Variable Forward swept Wing. Machinery and Electronics, 2011, 2011(4): 8-11.

[6].Liang J H, Liu H X, Shu X B. A 90-Deg-Alterable and Self-Lockable Mechanism for Variable Backward-Swept Wing: China, 2010 10116741. 2010.

[7]. Woldemar Voigt. Swept Wing with Unswept Span: USA, 3018985. 1962.

[8].Lu C W. World's Aircraft Handbook. Beijing: Aviation Industry Press, 1988: 30-163.

[9].Fang B R. Aircraft aerodynamic Configuration Design. Beijing: Aviation Industry Press, 1997: 363-367.

[10]. Chen P, Wang X, Hu M Q, Yu C. The Design of Wing-body Switching Mechanism with Two Slideways[J], ActaAeronauticaSinica, 2012,33(11),p.2010-2017. 
[11]. SU X B, Zhou Z, Wang X,SHI J C.The Analysis of Motion Characteristics of Variable Forward-swept Wing Mechanism with a Double Slideways[J],Research in Mechanical Engineering and Material Science,MME2013,November 22-24,2013,137-141. 